\title{
A spontaneous pregnancy and live birth in a woman with primary infertility following the excision of an ovarian adrenal rest tumor: A rare case
}

\author{
Hacer Uyanıkoglu', Gonul Ozer', Semra Kahraman² \\ 'Department of Obstetrics and Gynecology and IVF Unit, Harran University Medical Faculty, Sanliurfa; ${ }^{2}$ In Vitro Fertilization Unit, Istanbul Memorial \\ Hospital, Istanbul, Turkey
}

Adrenal rest tumors are a rare extra-adrenal complication of congenital adrenal hyperplasia (CAH) in women although they are more commonly found in the testes of male patients with CAH. An ovarian adrenal rest tumor (OART) may coexist with CAH or imitate its symptoms without CAH. In this case report, we present the case of a woman with OART without CAH, whose main complaint was infertility and who had a baby after successful surgical treatment.

Keywords: Infertility; Laparoscopy; Ovarian adrenal rest tumor

\section{Introduction}

Ovarian adrenal rest tumors (OARTs) occur in a very small proportion $(<0.1 \%)$ of women with congenital adrenal hyperplasia (CAH), and are extremely rare in women without $\mathrm{CAH}$ [1], although adrenal rest tumors are relatively common in the testes of men with CAH [2]. According to the literature, these tumors imitate CAH symptoms (e.g., hirsutism, clitoromegaly, oligo-amenorrhea, and, rarely, pelvic masses) [3]. Herein, we describe the first known case of OART in a woman without $\mathrm{CAH}$ presenting with primary infertility.

\section{Case report}

This is a case report, and the patient and her relatives were informed in detail and their written consents were obtained.

Received: March 24, 2020 • Revised: May 5, 2020 • Accepted: May 26, 2020 Corresponding author: Hacer Uyanıkoglu

Department of Obstetrics and Gynecology and IVF Unit,Harran University Medical Faculty, Sanliurfa, Turkey

Tel: +90-5355740425 Fax: +90-414-312-9785 E-mail: huoglu@hotmail.com

This is an Open Access article distributed under the terms of the Creative Commons Attribution Non-Commercial License (http://creativecommons.org/licenses/by-nc/4.0/) which permits unrestricted non-commercial use, distribution, and reproduction in any medium, provided the original work is properly cited.
A 34-year-old nulliparous woman presented to our in vitro fertilization (IVF) center because of an inability to conceive for 2 years. While taking her medical history, the patient reported that her menstrual cycle had started at the age of 13 , and was regular until the age of 19. Upon her first examination, the patient had been diagnosed with polycystic ovary syndrome due to her severe hirsutism and secondary amenorrhea (two of the three Rotterdam 2013 criteria). Therefore, she used oral contraceptive (OC) pills for 12 years. Her menstrual cycle improved while taking $\mathrm{OC}$ medication, and her hirsutism partially improved. She discontinued OC treatment after marriage because of a desire to have a child.

Two months after marriage, her irregular menses recurred. She presented to a local hospital with this complaint. Upon evaluation, her basal serum 17-hydroxyprogesterone (17-OHP) level was 20 $\mathrm{ng} / \mathrm{mL}$, which was higher than normal, leading the clinicians to consider the possibility of CAH. Therefore, dexamethasone therapy $(0.5$ $\mathrm{mg} /$ day) was started. Several ovulation inductions (with an aromatase inhibitor and timed coitus for six cycles) and an IVF cycle were performed with the goal of conception after the 6th month of her marriage. During IVF therapy, the gonadotropin (recombinant follicle- stimulating hormone [FSH]) dose used was $300 \mathrm{IU} /$ day for 9 days and the endometrial thickness was $6.4 \mathrm{~mm}$ on trigger day. From 
the two collected oocytes, one mature oocyte was obtained and fertilized. The embryo was transferred on day 3, but she failed to achieve a pregnancy, even though her husband was healthy and his semen analysis was normal.

When she was transferred to us, we performed a physical examination of the patient. We assessed hirsutism using the Ferriman-Gallwey score, with a score of 22. There were also signs of virilism, including thickening of the vocal cords, alopecia, and clitoromegaly. The results of the hormone assay indicated that the patient's 17-OHP level remained high $(86 \mathrm{ng} / \mathrm{mL})$, despite dexamethasone therapy. Her dehydroepiandrosterone sulfate, free testosterone, and total testosterone levels were $385.9 \mu \mathrm{g} / \mathrm{dL}, 5.5 \mathrm{pg} / \mathrm{mL}$, and $416.2 \mathrm{ng} / \mathrm{dL}$, respectively. Serum FSH, luteinizing hormone, thyroid-stimulating hormone, free T4, prolactin, and cortisol levels were normal. Transvaginal ultrasonography showed a normal uterus size, with a thin endometrium ( $4 \mathrm{~mm}$ ) and diminished ovarian reserve. The right ovary measured $40 \times 35 \times 32 \mathrm{~mm}$ in size and had one antral follicle, and the left ovary measured $29 \times 25 \times 25 \mathrm{~mm}$ in size and had three antral follicles. A $34 \times 30 \times 29 \mathrm{~mm}$ solid mass was observed in the right ovary (Figure 1). Tumor markers (i.e., CA 125, CA 15.3, alpha-fetoprotein, and carcinoembryonic antigen) were found to be within normal limits. Since she had an ovarian mass and a high serum 17-OHP level, we suspected an adrenal rest tumor and ordered an abdominal and pelvic magnetic resonance imaging scan. It showed a $42 \times 32-\mathrm{mm}$ hyperintense right ovarian solid mass with a normal left ovary; however, the bilateral adrenal glands were of normal size and morphology (Figure 2). We planned laparoscopy for a definitive diagnosis of the right ovarian mass. Laparoscopic examination of the pelvic cavity showed a yellowish solid $4-\mathrm{cm}$ mass on the right ovary. The mass was removed in an endoscopic bag without rupture (Figure 3), and sent for an immediate pathological frozen section, which revealed a benign tumor. Then it was sent for histological examination. Macroscopically, it was a yellow-colored tumor measuring nearly $4 \mathrm{~cm}$, with a smooth and well-limited surface. Microscopically, almost all of the
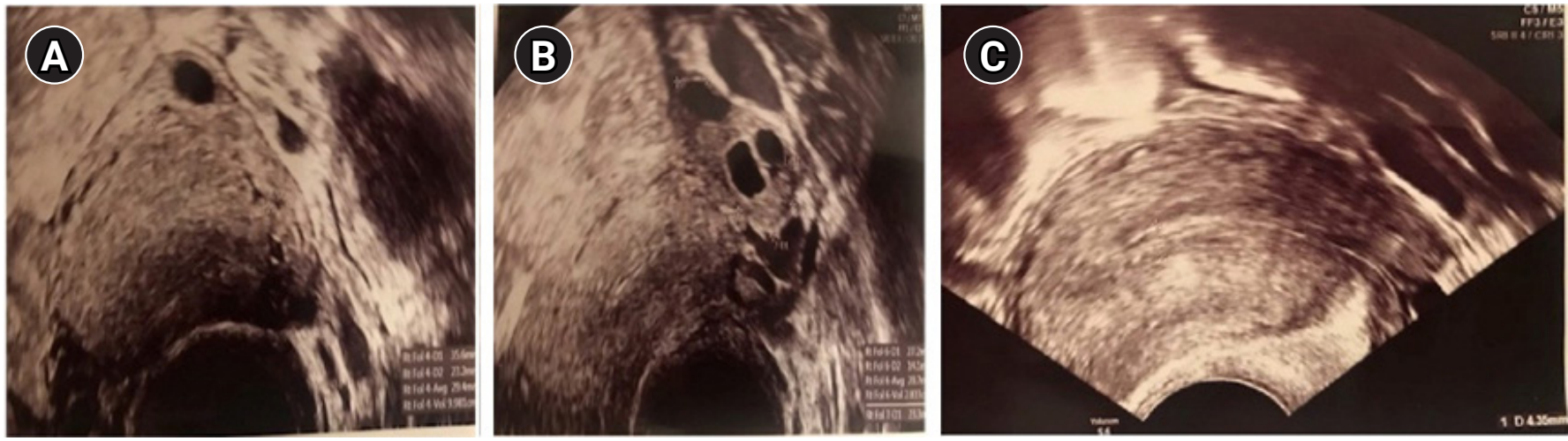

Figure 1. Ultrasonographic appearance of the right ovarian adrenal rest tumor demonstrating an echogenic border (A), the left ovary (B), and thin endometrium (C).

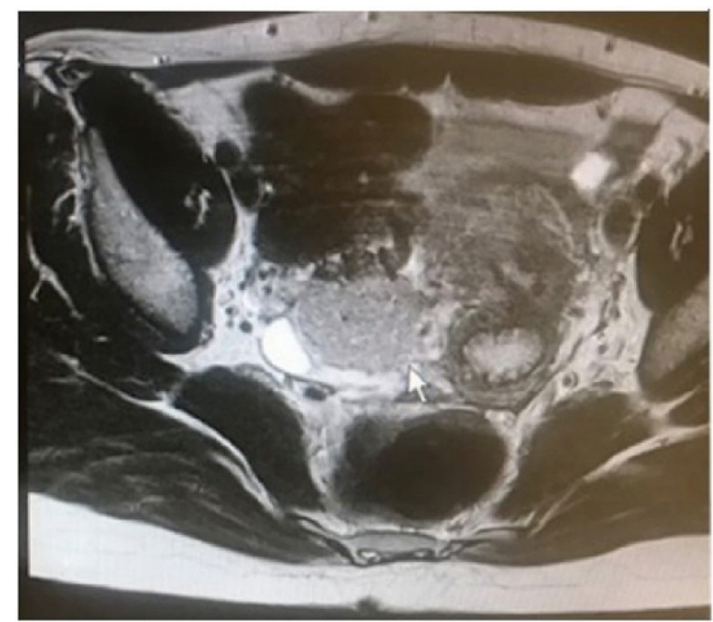

Figure 2. Magnetic resonance imaging showed a $42 \times 32-\mathrm{mm}$ hyperintense right ovarian solid mass in the pelvic cavity.

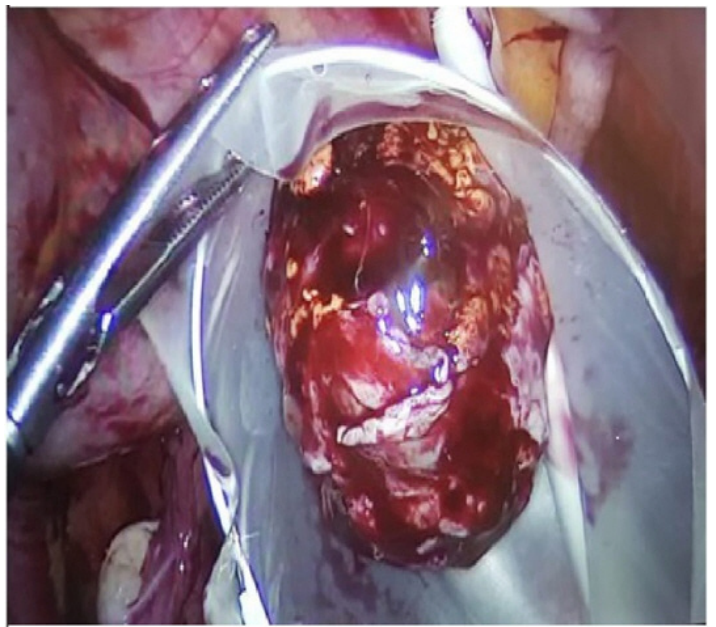

Figure 3. Laparoscopic removal of the yellowish solid mass in an endoscopic bag. 
ovarian parenchyma was occupied by the tumor lesion, as shown by large cells with small nuclei without mitotic activity, nuclear atypia, and Reinke crystalloids. Hemorrhagic or necrotic areas were absent in the sample. Immunohistochemical analysis revealed diffuse staining for calretinin, inhibin, vimentin, and melan-A (Figure 4). The final histopathological findings confirmed the diagnosis of OART.

One week after the operation, the patient's serum 17-OHP level decreased dramatically (1.82 ng/mL). Therefore, we suspected CAH, and consulted with an endocrinologist to carry out an adrenocorticotropic hormone (ACTH) simulation (Synacthen) test for the definitive diagnosis of $\mathrm{CAH}$. In the ACTH simulation test, the 17-OHP levels were $0.21 \mathrm{ng} / \mathrm{mL}, 0.54 \mathrm{ng} / \mathrm{mL}$ and $0.60 \mathrm{ng} / \mathrm{mL}$ at 0,30 , and $60 \mathrm{~min}$ utes, respectively. Based on these findings, the diagnosis of $\mathrm{CAH}$ was excluded. We also conducted a genetic test to investigate the possible presence of a CYP21A2 gene mutation, the test result was negative. We offered detailed genetic tests for the other enzyme deficiencies involved in $\mathrm{CAH}$, but the patient did not agree to undergo the recommended tests.

Postoperatively, the patient's menstrual cycles became regular and signs of hyperandrogenism disappeared. Furthermore, 4 months after the operation, she spontaneously became pregnant and then gave birth to a healthy infant at term.

\section{Discussion}

Adrenal rest tumors are benign, but may produce excessive 17$\mathrm{OHP}$ and androgens. These tumors are commonly found in the testes of male patients, and their prevalence has been reported to be as high as $95 \%$ in men with CAH. In contrast, OARTs are less common in women with $\mathrm{CAH}(<0.1 \%)$ [1]. In a literature search, we found that

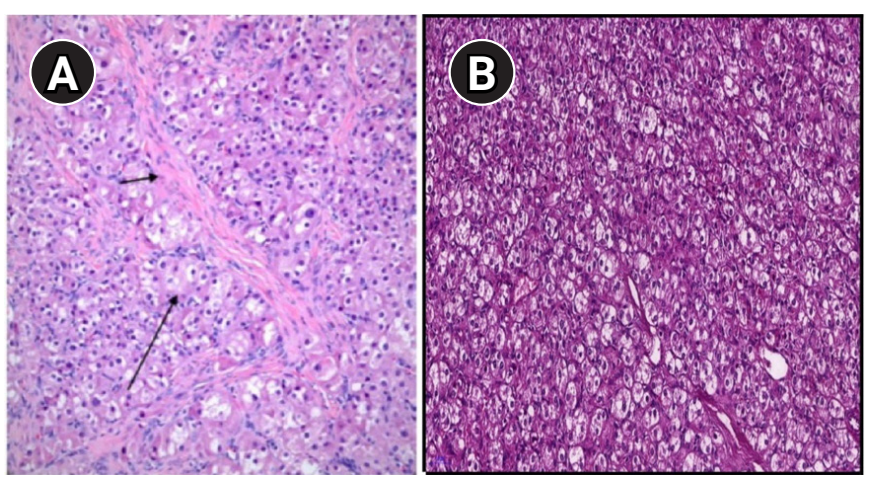

Figure 4. (A) Microscopic appearance of the tumor cells showed a uniform size, polygonal shape, abundant eosinophilic and vacuolated cytoplasm, and round nuclei with small nucleoli (long arrow). Acellular hyaline areas were observed among clusters of tumor cells (short arrow). (B) Mitotic activity was low. Crystalloids of Reinke were not identified $(\mathrm{H} \& \mathrm{E}, \times 200)$.
Yilmaz-Agladioglu et al. [3] reported the first case of OART in a girl without $\mathrm{CAH}$. They presented a 13-year-old female patient who was diagnosed with non-classical $\mathrm{CAH}$ at 6 years of age due to premature pubarche, and received hydrocortisone therapy for 6 years. The patient was further investigated because hormonal control was unsuccessful (high levels of 17-OHP and total testosterone) while under high-dose steroid therapy, and she was diagnosed with a steroid cell ovarian tumor, similar to our case. In our case, signs of hyperandrogenism, disordered menstruation, and infertility were the main complaints of the patient, whereas, in their case, the patient's complaint was limited to signs of hyperandrogenism. This discrepancy reflects the fact that the patients were at different reproductive stages. Surgical removal of the ovarian mass resulted in clinical improvements in both cases. To the best of our knowledges, our patient is second case in which OART was seen in a woman without $\mathrm{CAH}$.

The mechanism of ART formation still has not yet been fully elucidated. Kim et al. [4] mentioned that these tumors may arise from ectopic migration of residual adrenal cortex cells into the developing gonads because of the connection between the adrenal glands and gonads during embryonic development. Claahsen-van der Grinten et al. [5] suggested that defective primary sex cord regression, including all aberrant adrenal cells, during gonad development may be a causative factor in OART development.

In the literature, it has been shown that OARTs were related with distinct enzyme deficiencies associated with $\mathrm{CAH}$, such as 21-hydroxylase, 11-hydroxylase, and 3- $\beta$ hydroxysteroid dehydrogenase [6]. In our case, beside the negative results of Synacthen and genetic (CYP21A2 gene) tests, improvement of the patient's clinical complaints (menstrual disturbances and hyperandrogenism signs, even including infertility) after the operation, supported exclusion of the diagnosis of $\mathrm{CAH}$.

The diagnosis of OART masses by conventional imaging may be difficult because of the rarity of OART and sometimes small size of these tumors. Chen et al. [7] recommended examination of the ovaries and excision of any suspicious OART masses in female CAH patients who respond poorly to hormone therapy. In our case, we also suspected OART because the patient was unresponsive to hormone therapy and because of the patient's ongoing complaints; additionally, the patient stated that this ovarian mass had been present for a long time, but it was not considered important since it was small.

Testicular adrenal rest tumors may be an important cause of infertility in men, as they lead to obstruction of the seminiferous tubules, azoospermia and gonadal dysfunction [8]. Disruption in the hypothalamic-pituitary-ovarian axis due to excessive androgens and excessive progesterone secretion in OART patients can causes infertility. Androgens can also directly inhibit folliculogenesis by having a negative effect on aromatase activity in granulosa cells. Additionally, 
progesterone hypersecretion in OARTs can affect the quality of cervical mucus and sperm penetration, accelerate endometrial maturation, reduce endometrial receptivity, decrease implantation, and exert negative effects on oocyte quality [9].

To the best of our knowledge, this is the first known case of OART causing infertility. There are three principal aspects that should be noted: first, this was an extremely rare case; second, the patient's infertility and other hyperandrogenism-related complaints were successfully treated; and third, it was possible to exclude the diagnosis of $\mathrm{CAH}$, which requires excessive steroid use and repeated treatments for infertility. Increased levels of androgen hormones by OARTs may lead to infertility by damaging the hypothalamo-hypophyseal axis. The appropriate diagnosis and treatment of these cases can prevent both excessive steroid use and recurrent treatments for infertility. The patient described herein conceived spontaneously following the excision of an OART.

\section{Conflict of interest}

No potential conflict of interest relevant to this article was reported.

\section{ORCID}

Hacer Uyanıkoglu https://orcid.org/0000-0003-0316-4900

\section{Author contributions}

Conceptualization: SK. Data curation: GO, HU. Formal analysis: HU. Funding acquisition: SK. Methodology: GO. Project administration: SK. Visualization: HU. Writing-original draft: HU. Writing-review \& editing: HU.

\section{References}

1. Stikkelbroeck NM, Hermus AR, Schouten D, Suliman HM, Jager GJ, Braat DD, et al. Prevalence of ovarian adrenal rest tumours and polycystic ovaries in females with congenital adrenal hyperplasia: results of ultrasonography and MR imaging. Eur Radiol 2004;14: 1802-6.

2. Osaka A, Ide H, Ono Y, Arai G, Ueda Y, Okada H. Testicular adrenal rest tumor without congenital adrenal hyperplasia: a rare case. Int J Urol 2018;25:903-4.

3. Yilmaz-Agladioglu S, Savas-Erdeve S, Boduroglu E, Onder A, Karaman I, Cetinkaya S, et al. A girl with steroid cell ovarian tumor misdiagnosed as non-classical congenital adrenal hyperplasia. Turk J Pediatr 2013;55:443-6.

4. Kim MS, Koppin CM, Mohan P, Goodarzian F, Ross HM, Geffner $M E$, et al. Absence of testicular adrenal rest tumors in newborns, infants, and toddlers with classical congenital adrenal hyperplasia. Horm Res Paediatr 2019;92:157-61.

5. Claahsen-van der Grinten HL, Otten BJ, Stikkelbroeck MM, Sweep FC, Hermus AR. testicular adrenal rest tumours in congenital adrenal hyperplasia. Best Pract Res Clin Endocrinol Metab 2009;23: 209-20.

6. Zaarour MG, Atallah DM, Trak-Smayra VE, Halaby GH. Bilateral ovary adrenal rest tumor in a congenital adrenal hyperplasia following adrenalectomy. Endocr Pract 2014;20:e69-74.

7. Chen HD, Huang LE, Zhong ZH, Su Z, Jiang H, Zeng J, et al. Ovarian adrenal rest tumors undetected by imaging studies and identified at surgery in three females with congenital adrenal hyperplasia unresponsive to increased hormone therapy dosage. Endocr Pathol 2017;28:146-51.

8. Karen Leong SW, Wu LL. Case series of testicular adrenal rest tumours in boys with congenital adrenal hyperplasia: a single centre experience. Med J Malaysia 2019;74:92-3.

9. Gomes LG, Bachega TA, Mendonca BB. Classic congenital adrenal hyperplasia and its impact on reproduction. Fertil Steril 2019; 111:7-12. 